\title{
Information Embedding in Additive Manufacturing through Printing Speed Control
}

\author{
Karim A. ElSayed \\ kelsayed@purdue.edu \\ Purdue University \\ West Lafayette, Indiana, USA
}

\author{
Adam Dachowicz \\ adachowi@purdue.edu \\ Purdue University \\ West Lafayette, Indiana, USA
}

\author{
Jitesh H. Panchal \\ panchal@purdue.edu \\ Purdue University \\ West Lafayette, Indiana, USA
}

\begin{abstract}
Additive manufacturing (AM) is rapidly developing, and new applications are continuously emerging. While AM is increasingly becoming integral to many industries, including aerospace, automotive, and biomedical, it has opened a host of unique security concerns, from theft of technical data to process sabotage and counterfeiting. In this work, we present a method to address the counterfeiting problem by embedding information in additively manufactured parts through controlling printing process parameters. Variations in printing speed, the encoding parameter in this work, introduce subtle localized height differences on parts' surfaces, which are readable using an optical profilometer. The profilometry data is captured after printing, and this data is processed to predict the intended bit response for each embedding region on the surface of the part. We experimentally demonstrate the feasibility of the proposed scheme for embedding and reading the information in $3 \mathrm{D}$ printed parts and show that it achieves $80 \%$ accuracy for a $53 \mathrm{~mm} / \mathrm{s}$ difference in printing speed between the encoded bits. Finally, we characterize the performance of the proposed scheme, measured as the accuracy in decoded messages, as a function of the difference in printing speed used to perform the embedding.
\end{abstract}

\section{CCS CONCEPTS}

- Security and privacy $\rightarrow$ Security in hardware.

\section{KEYWORDS}

Information Embedding; Additive Manufacturing; Anti-counterfeiting; Information Capacity

\section{ACM Reference Format:}

Karim A. ElSayed, Adam Dachowicz, and Jitesh H. Panchal. 2021. Information Embedding in Additive Manufacturing through Printing Speed Control. In Proceedings of the 2021 Workshop on Additive Manufacturing (3D Printing) Security (AMSec '21), November 19, 2021, Virtual Event, Republic of Korea. ACM, New York, NY, USA, 7 pages. https://doi.org/10.1145/3462223.3485623

\section{INTRODUCTION}

Additive manufacturing (AM), also known as 3D printing, has seen rapid growth in the past few decades. It offers significantly greater

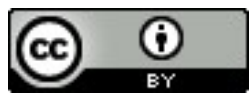

This work is licensed under a Creative Commons Attribution International 4.0 License. AMSec '21, November 19, 2021, Virtual Event, Republic of Korea. (c) 2021 Copyright is held by the owner/author(s). ACM ISBN 978-1-4503-8480-3/21/11.

https://doi.org/10.1145/3462223.3485623 control over geometric features and material properties of manufactured goods compared to competing subtractive manufacturing methods, and advances in AM have disrupted several key industries, including aerospace, automobiles, and bio-medicine [3, 11, 14]. While AM has driven rapid progress in these areas, it has also opened a host of unique security concerns, from theft of technical data to process sabotage and counterfeiting [27]. One approach to addressing traceability in general, and anti-counterfeiting in particular, is to embed information in additively manufactured parts so end users can verify their origin.

Existing literature in information embedding in AM presents methods to embed and read information from 3D printed parts, for a range of printing processes and embedded signals. These signals include acoustic responses, cavities in the part volume, material changes, and others $[8,20,24]$. Embedding these signals requires manipulation of parts' geometry or printing process parameters (material extrusion, print head path, etc.) to locally alter parts to carry the desired signal. In this work, we propose a different scheme for embedding information on the surface of additively manufactured parts. This scheme embeds information by manipulating printing speed to create subtle differences in the surface characteristics that can be measured by optical profilometry. We also investigate the accuracy of proposed decoding methods as a function of the variation of the encoding parameter, i.e., the printing speed.

This paper is structured as follows: Section 2 provides an overview of related work and discusses embedding from an information theory prospective. In Section 3, we explain our approach and the experimental setup used for information embedding and detection. We present and discuss our results in Section 4. Finally, we conclude our paper and discuss future work in Section 5.

\section{BACKGROUND}

Due to the rapid growth and adoption of AM technologies, the risk of counterfeiting AM-fabricated components is rising [2, 27]. Many researchers have aimed at addressing this risk [2, 4, 13, 20, 27]; however, the research in this field is still relatively new [5]. In this section, we review the literature to highlight various approaches used to embed information in AM-fabricated components. All the methods discussed use polymer-based AM techniques, unless otherwise specified.

\subsection{Information Embedding in AM}

Through AM, information can be embedded on a physical part's surface or as part of its internal geometry. Several means of embedding include: modifying parts' geometry $[8,17,28]$, manipulating printing process parameters $[5,7,9]$, and incorporating multiple materials (filaments or feedstock) $[16,24]$. Depending on the method, 
information may be embedded in different locations on or in a part. Each embedding location has its own pros and cons. Surface-level information is generally simple and low-cost to capture, requiring equipment such as cameras [7], scanners [5], or microscopes. Conversely, obtaining internally embedded information may require more costly equipment, such as a Terahertz camera [26] or a micro-computed tomography (micro-CT) scanner [2]. However, the information on a part's surface may be less robust to withstand damage than that embedded inside a part.

2.1.1 Surface Embedding. Aliaga and Atallah [1] presented one of the earlier works addressing the counterfeiting problem in AM. Their approach injects subtle geometric noise on the surface that, in combination with the inherent manufacturing noise, is used as a signature of the part; however, detecting it is challenging without a good estimation of the noise in the manufacturing process. Other more obtrusive approaches explore modifying the part's geometry to include $3 \mathrm{D} \mathrm{QR}$ (or similar to $\mathrm{QR}$ ) codes on the surface $[19,28]$. Peng et al. [17] embed less obtrusive QR codes through introducing minimal carvings on the surface geometry, but a directional light source with a predefined angle is needed for successful detection. Acoustic Barcodes [8] create surface notches that generate a desired sound when swiped, which is captured by a microphone and mapped to a barcode. LayerCode [16] also embeds barcode patterns on parts' surfaces, however, by either changing layer color (for multi-extruder printers) or height of alternating layers to signify the two binary bits ("0" and " 1 ") in a barcode.

Most similar to our approach are embedding techniques that utilize printing process parameters. For example, $G-I D$ [7] creates unique signatures on the surface (or near-surface) of parts through exploiting possible variations in process parameters (e.g., infill pattern, density, and layer height). Delmotte et al. [5] embed binary bit-strings by varying the layer height locally (i.e. across the same layer). This enables the encoding of large amount of information with a small footprint.

2.1.2 Internal Embedding. Kubo et al. first proposed FabAuth [10], a method that manually modifies the internal geometry of a part to create unique patterns identifiable by ultrasonic sensing. Then, they extended this approach to embed using process parameter variations (e.g., infill pattern or density), instead of manually changing the internal structure [9]. 3D QR codes have also been embedded inside printed parts using both polymer-based [2] and metalbased [2, 24] AM machines; however, their detection requires the use of a micro-CT scanner [2] or an X-ray machine [24]. Other researchers explored placing air voids below the outer layers of parts as a way of embedding information [12, 20,26]. Though successful, they may require special equipment for detection, such as an infrared (IR) camera [20] or a Terahertz imaging system [26]. Additionally, the existence of air gaps could compromise the integrity of the parts, potentially making them unfit for some load-bearing applications.

\subsection{Embedding from a Coding Theory Perspective}

The fields of information theory and coding theory offer methods to analyze the quality of information embedding schemes in a quantifiable way. Mackay and coauthors [15] present a thorough introduction to information theory and the practical applications of coding theory in machine learning and communications tasks. Of particular relevance here is channel coding, which involves developing methods to transmit messages across some channel, such as a telephone line. We consider here the problem of communicating over a noisy channel: we assume some message $m$ (e.g. natural text or part identifier) is encoded as $x$ (a bit string), and the encoded message passed through a channel where some noise process corrupts the encoded data. Then, the corrupted encoding $\hat{x}$ is decoded by the receiver to yield a possibly corrupted message $\hat{m}$. This is illustrated in Figure 1. Error correcting codes such as block codes [25], convolutional codes [23], or turbo codes [6] may be implemented during encoding and decoding to better recover the intended message at the cost of reducing the information rate, or the information transmitted per unit time.

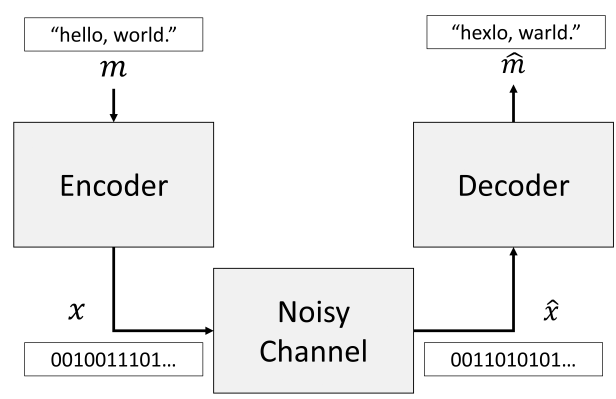

Figure 1: Illustration of encoding and decoding over a noisy channel.

In this paper, we present an encoding method where one manufacturing process parameter is varied (printing speed) to encode the bit string on the surface of a part. Height data acquired during decoding is used to recover the message, and noise is assumed to be introduced through the manufacturing and data acquisition processes.

\section{APPROACH AND EXPERIMENTAL SETUP}

Designing an information embedding scheme for manufacturing can be cast as the design of an encoder that writes information to the surface of a part through some process, and the corresponding decoder that reads that information when necessary. The information is passed through a channel that consists of all processes impacting the encoded information from the time of encoding (manufacturing) to decoding by the end user. Our task is to design an encoding scheme that maximizes the amount of information that can be transmitted in the part (the channel capacity) given constraints placed on the channel by the manufacturing process, part design requirements, expected damage to the part, and available data acquisition methods. Design variables for this channel may include the specific manufacturing process parameters to vary, the range over which those parameters are allowed to vary, the size 


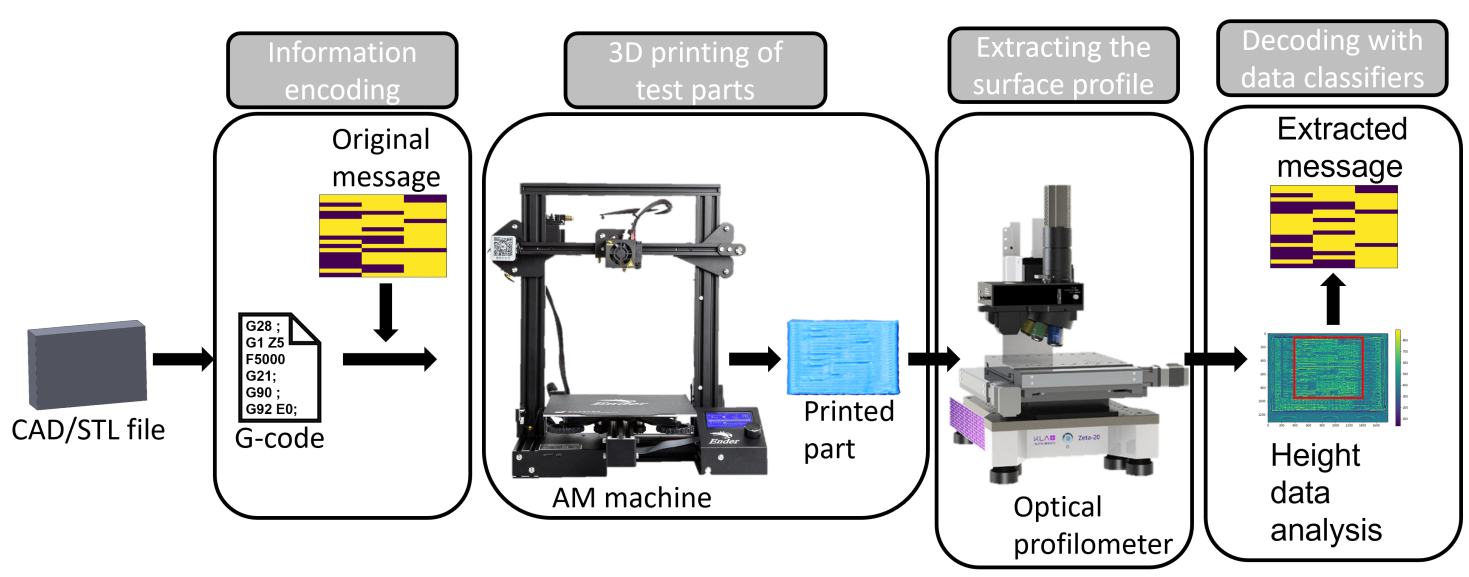

Figure 2: Embedding and reading information pipeline.

of the manipulated regions of the part, and the part characteristics that are measured during decoding. The manufacturer should also characterize the noise of the channel, which may be due to inaccuracies during encoding in manufacturing, damage to the part after manufacturing but before decoding, noise introduced during data acquisition during decoding, or some combination of all these factors.

In this paper, we propose an embedding scheme that embeds information in AM parts through controlling the printing process parameters. Specifically, we chose the printing speed as our encoding parameter for this work. The channel is the surface modified during printing, and decoding is done by processing height measurements of this surface to read the embedded message, given any noise that might have been introduced during manufacturing, handling, and data collection. In this section, we present our encoding scheme, experimental and data collection setup, and decoding methodology for reading the embedded data.

\subsection{Encoding, Printing, and Imaging}

In this work, we begin by generating a binary string of bits containing the desired message. These bits are mapped to a region on the surface of the 3D model, without any manipulation of it. Then, in the locations where the bits are to be embedded, we modify the printing speed in the model's g-code, such that the printing speed for a " 0 " bit is different from that of a " 1 " bit. After printing, we obtain the surface profile data (i.e., height data) from the 3D part using an optical profiling microscope and analyze it to decode the embedded message. This overall experimental pipeline is shown in Figure 2.

In the following sections, we discuss the encoding scheme and the experimental conditions for 3D printing the test parts and collecting their height data in more detail.

3.1.1 Information Encoding. To embed an $n$-bit message, we first reshape the binary bit string into an array corresponding to the area on the surface of the part that would encode bits. The array consists of $R$ rows and $C$ columns, where $R \times C=n$. For example, a 28 bit string consisting of alternating " 1 " and " 0 " is embedded using a $1 \mathrm{D}$ array with $R=28$ and $C=1$. Figure 3 displays this $1 \mathrm{D}$ alternating array, the raw image of the encoded surface, and the preprocessed height data.

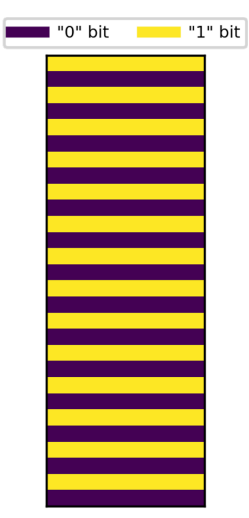

(a)

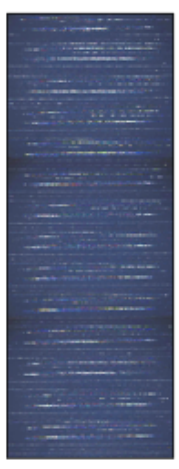

(b)

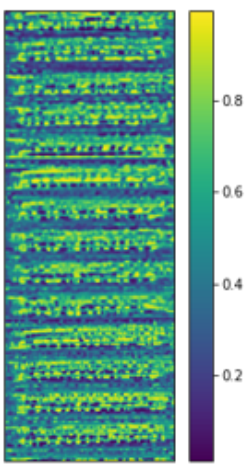

(c)
Figure 3: Different representation of the encoded region used for training the classifiers: (a) 1D embedded bit string, (b) raw image, (c) normalized height data.

Given a bit string that is reshaped into a $R \times C$ array, we map it onto an area on the surface of the part. First, we define the maximum information capacity of the surface, which is inversely proportional to the width of a bit, $b_{w}$, number of layers in a bit, $b_{\text {num }}$, and layer height, $h$. In general, the surface area needed to encode $R \times C$ bits is:

$$
A_{\text {encode }}=R \cdot C \cdot b_{w} \cdot b_{\text {num_l }} l \cdot h .
$$

Ideally, one prefers to have as small of an $A_{\text {encode }}$ as possible for encoding $n$ bits (i.e., high information capacity) to minimize the effect of information embedding on the surface quality. However, doing so complicates the extraction of bits, increasing the chances of misclassifying them. In this work, we tested our method to encode 60 random bits reshaped as a $20 \times 3$ array, as shown in Figure 4, on 
three identical parts using different encoding speeds with $b_{w}=2.4$ $\mathrm{mm}$ and $b_{\text {num }} l=2$ layers. In the following section, we outline the process parameters used in printing the physical parts with the encoded 60 bit message.

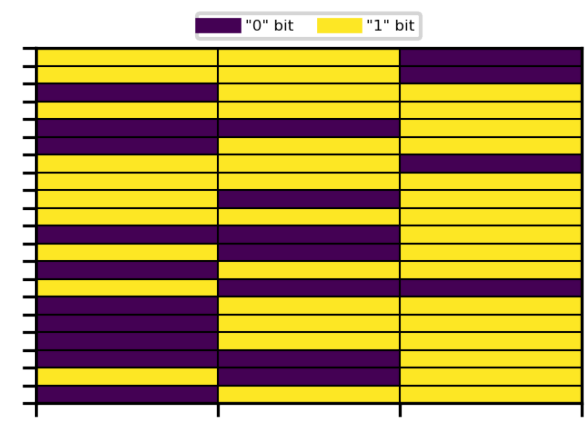

Figure 4: Test message.

3.1.2 3D Printing of Test Parts. To assess the feasibility of the proposed information embedding and extracting method, we printed three parts with the 60 bit encoded message using Creality Ender $3,{ }^{1}$ a commercial fused deposition modeling (FDM) 3D printer. All parts used the same $8 \times 12 \times 3.6 \mathrm{~mm}$ rectangular CAD model, and their g-code was generated using Slic3r open-source software. ${ }^{2}$ They were printed using a PLA filament and the original g-code file was generated using the following printing parameters: 0.16 mm layer height $(h), 200^{\circ} \mathrm{C}$ nozzle temperature, $100 \%$ infill density, $60 \mathrm{~mm} / \mathrm{sec}$ infill speed, and $30 \mathrm{~mm} / \mathrm{sec}$ external perimeter speed.

Although the test parts have the same printing parameters, we varied the bit encoding speed among them to investigate how the difference in speed, $\Delta s$, between the " 0 " and " 1 " bits affects our embedding and extraction scheme. Specifically, we fixed the speed for embedding the "0" bits and used three different speeds for encoding the " 1 " bits. Table 1 shows the bit-encoding speeds, with the highest and lowest speed differences used in Part 1 and Part 3, respectively.

Table 1: Bit encoding speeds.

\begin{tabular}{|c|c|c|c|}
\hline Part number & Part 1 & Part 2 & Part 3 \\
\hline “0” bit speed (mm/s) & 60 & 60 & 60 \\
\hline “1” bit speed (mm/s) & 7 & 28 & 50 \\
\hline$\Delta \mathbf{s ~ ( m m / s ) ~}$ & 53 & 32 & 10 \\
\hline
\end{tabular}

3.1.3 Extracting the Surface Profile. For all parts, we obtained the height data signals of the entire front surface using Zeta-20 optical profiling microscope. ${ }^{3}$ We used the $5 x$ lens, which has a field of view $(\mathrm{FoV})$ of $4.69 \mathrm{~mm} \times 3.52 \mathrm{~mm}$. Given this limited FoV for our application, we took nine scans covering each of our parts and stitched them using MATLAB DIPimage 2.9 [22]. An example of the resulting height map is shown in Figure 3c.

${ }^{1}$ Brochure information available: https://www.creality.com/goods-detail/ender-3-3dprinter

${ }^{2}$ Available: https://slic3r.org/

${ }^{3}$ Brochure information available:

tencor.com/products/instruments/optical-profilers/zeta-20

\subsection{Decoding with Data Classifiers}

Given the surface height data for a part, we now must design a method to determine whether a given region of the surface is encoding a "0" or " 1 " bit. We present results for two classifiers: line-based and area-based. For both, we use the preprocessed height data, represented as an image with pixels of intensity proportional to the original height data. The first classifies each horizontal line of pixels as coming from a " 0 " or " 1 " region based on the mean intensity of the pixels along the line. The second performs a similar classification but considers the mean intensity in a $2 \mathrm{D}$ region extending above and below each horizontal line. We assume we know a priori which regions of the surface encode bits, and further that we can divide the image into the regions where each bit is embedded. In practice, this assumption can be justified by asserting that all embedded messages are applied to identical regions of a part's surface, and carry bit information in some regular pattern on the surface.

3.2.1 Height Data Preprocessing. All classifiers discussed in this section take as input the surface height data. This data is equalized using the Contrast Limited Adaptive Histogram Equalization (CLAHE) algorithm [18] to better capture local changes in height, with contextual region equal to $1 / 8$ image height by $1 / 8$ image width. The scikit-image implementation of the CLAHE algorithm was used for this study [21]. The images are then blurred using a Gaussian filter with standard deviation of 7 pixels to remove high-frequency noise from the image.

3.2.2 Training Data. The classifiers discussed in this section used the same printed part as training data. A height image of this part is provided in Figure 3c. Details on the data from this image used for training are provided for each classifier below.

3.2.3 Line-Based Classifier. A total of 42 horizontal line samples are extracted from regions in the equalized training image corresponding to "0" and " 1 " bits each. The average intensities are taken for each of these samples, and the results are fitted to a normal distribution. Call the mean and standard deviation of the " 0 " and " 1 " samples $\left(\mu_{0}, \sigma_{0}\right)$ and $\left(\mu_{1}, \sigma_{1}\right)$, respectively. Given these statistics, at test time we compute the likelihood of any sample coming from a " 0 " or " 1 " region, and classify the sample as " 0 " if the 0 likelihood is greater, or else classify the sample as 1 . The ratio of these two likelihoods may be used as a measure of confidence in the classification (if close to 1, then bits are distinguished with less certainty), however in this study we do not use this information. At test time, classification is performed for all horizontal lines of pixels in each bit region (in this study, 23 samples were taken per bit), and the mode of the resulting classifications is used as the final prediction for that bit. In this way, each line contributes a "vote" and the majority decides the final classification.

3.2.4 Area-Based Classifier. The area-based classifier is a generalization of the line-based classifier where a "window" of $n$ lines above and below each sample location is taken, and the mean pixel value in the window is used to train the classifier and generate data at test time. If $n=0$, this reduces to the line-based classifier discussed above. The window size $n$ is a parameter that should be 
tuned for the given use case. In this paper, $n=10$ was used, meaning that each "vote" was computed using a window of $2 n+1=21$ lines extracted from a given bit region. We observed that $n$ values between 8 and 15 produced similar accuracy results. 14 samples each are extracted from " 0 " and " 1 " regions in the equalized training image, each representing the average intensity of a full bit region.

In the following section, we present and discuss the classification results of the encoded message for all the test parts.

\section{RESULTS}

We encoded the $20 \times 3$ array shown in Figure 4 on the parts' surfaces while printing using $b_{w}=2.4 \mathrm{~mm}, h=0.16 \mathrm{~mm}$, and $b_{\text {num_ } l}=$ 2 layers. Thus, the bit string is encoded in three vertical sections across forty layers. The total area of the encoded region is $A_{\text {encode }}=$ $7.2 \times 6.4 \mathrm{~mm}=46.08 \mathrm{~mm}^{2}$, which is less than half the area of front of the part. To simplify the detection of bits and reduce noise, we avoided encoding the message too close to the bottom or borders of the part. Figure 5 displays Part 1 with the encoded region in red.

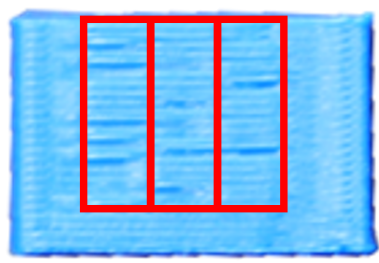

Figure 5: Part 1 with the encoded region boxed in red.

To investigate the effect of the encoding parameter on the accuracy of our decoding methods, we varied the speeds for embedding the " 1 " bits across parts according to the values reported in Table 1 . The resulting height maps after scanning, stitching, and preprocessing the data are shown in Figure 6. The encoded region of Part 1 showed more pronounced height differences than the other two parts. This can be attributed to its slower " 1 " encoding speeds (7 compared to 28 or $50 \mathrm{~mm} / \mathrm{sec}$ ), which indirectly causes more material to be deposited that results in height differences in the out-of-page direction.

We then identified the encoded regions and classified them using both the line-based and area-based classifiers discussed in Section 3.2. In Figures 7-9, we show the extracted and decoded messages for the three parts. As expected, the number of misclassified bits increases as the difference between " 0 " and " 1 " bit encoding speeds $(\Delta s)$ decreases. This is because as $\Delta s$ decreases, so does the height difference between the " 0 " and " 1 " bits.

Interestingly, both classifiers performed equally well on each part, though they misclassified bits in different locations. In Table 2, we report their performance on each part using confusion matrices. Out of 60 , the number of misclassified bits is 12,24 , and 29 for Parts 1, 2 and 3, respectively. Thus, the classification accuracy is $80 \%$ for Part 1, 60\% for Part 2, and 52\% for Part 3. In general, both classifiers were better at detecting " 1 " bits than "0" bits. One solution to reduce this disparity could include increasing the sample size used for training and testing the classifiers, or classifying using more features, e.g., height image data texture descriptors as in [13].

\section{CONCLUSION}

In this work, we presented a scheme for encoding information on the surface of additively manufactured parts by locally varying the printing speed. The information is decoded by reading height data gathered from the part's surface and processing this data to reconstruct the embedded message. Although the number of parts used to test this scheme was limited to three, the results show that (i) the printing speed can be used as an encoding parameter, and (ii) the accuracy of our decoding methods can be represented as a function of the encoding parameter.

As shown in Table 2, the performance of this scheme decreases as the difference in the encoding speed $(\Delta s)$ decreases. This result highlights a trade-off between the accuracy of the scheme and the imperceptibility of the surface features used to embed the message, which may be desired for anti-counterfeiting, functional or aesthetic reasons. It also motivates future work in the design of embedding schemes that optimize information carrying capacity subject to constraints imposed by the manufacturing process, use case, or other factors relevant to the manufacturer.

Our results show that embedding 60 bits in $0.46 \mathrm{~cm}^{2}$ is our maximum information capacity, using the current process and embedding parameters. In future work, our embedding method can be refined to both optimize the surface's information carrying capacity and increase the decoding accuracy. These can be achieved by carrying out more studies to optimize the encoding parameter, signal width, number of layers per signal, and layer height. Additionally, this scheme could be extended to encode more complex symbols that can carry more than one bit of information in each cell, further increasing information capacity, and include multiple encoding parameters (e.g., layer height, choice of material, and so on).

\section{REFERENCES}

[1] Daniel G Aliaga and Mikhail J Atallah. 2009. Genuinity signatures: Designing signatures for verifying 3d object genuinity. In Computer Graphics Forum, Vol. 28. Wiley Online Library, 437-446.

[2] Fei Chen, Yuxi Luo, Nektarios Georgios Tsoutsos, Michail Maniatakos, Khaled Shahin, and Nikhil Gupta. 2019. Embedding tracking codes in additive manufactured parts for product authentication. Advanced Engineering Materials 21, 4 (2019), 1800495.

[3] Lian Chen, Yong He, Yingxin Yang, Shiwei Niu, and Haitao Ren. 2017. The research status and development trend of additive manufacturing technology. The International fournal of Advanced Manufacturing Technology 89, 9-12 (2017), 3651-3660.

[4] Arnaud Delmotte, Kenichiro Tanaka, Hiroyuki Kubo, Takuya Funatomi, and Yasuhiro Mukaigawa. 2018. Blind watermarking for 3-d printed objects using surface norm distribution. In 2018 foint 7th International Conference on Informatics, Electronics \& Vision (ICIEV) and 2018 2nd International Conference on Imaging, Vision \& Pattern Recognition (icIVPR). IEEE, 282-288.

[5] Arnaud Delmotte, Kenichiro Tanaka, Hiroyuki Kubo, Takuya Funatomi, and Yasuhiro Mukaigawa. 2019. Blind watermarking for 3-D printed objects by locally modifying layer thickness. IEEE Transactions on Multimedia 22, 11 (2019), 2780-2791.

[6] Dariush Divsalar and Fabrizio Pollara. 1995. On the design of turbo codes. The Telecommunications and Data Acquisition Progress Report 42-123 (1995).

[7] Mustafa Doga Dogan, Faraz Faruqi, Andrew Day Churchill, Kenneth Friedman, Leon Cheng, Sriram Subramanian, and Stefanie Mueller. 2020. G-ID: Identifying 3D Prints Using Slicing Parameters. In Proceedings of the 2020 CHI Conference on Human Factors in Computing Systems. 1-13.

[8] Chris Harrison, Robert Xiao, and Scott Hudson. 2012. Acoustic barcodes: passive, durable and inexpensive notched identification tags. In Proceedings of the 25th annual ACM symposium on User interface software and technology. 563-568.

[9] Yuki Kubo, Kana Eguchi, and Ryosuke Aoki. 2020. 3D-Printed Object Identification Method using Inner Structure Patterns Configured by Slicer Software. In Extended Abstracts of the 2020 CHI Conference on Human Factors in Computing Systems. 1-7. 


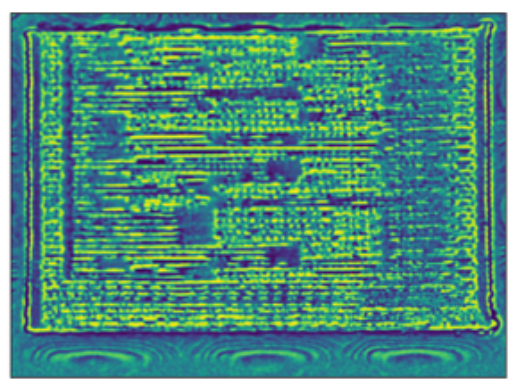

(a)

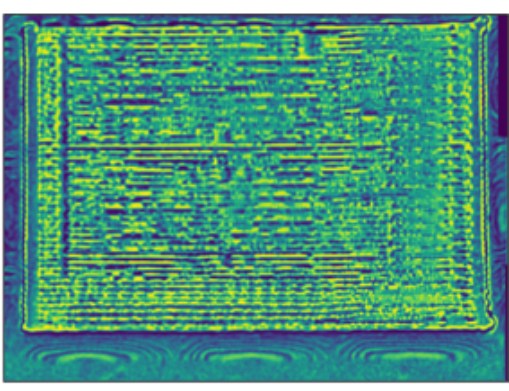

(b)

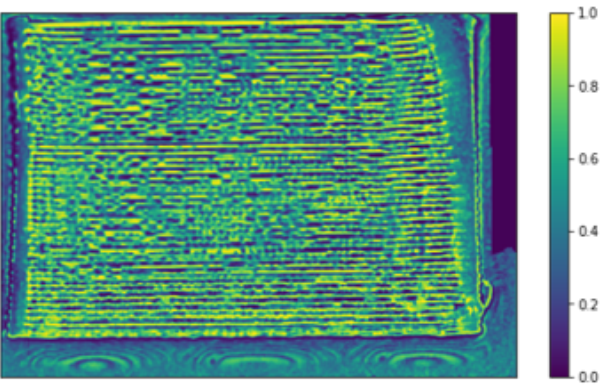

(c)

Figure 6: Equalized height maps for (a) Part 1, (b) Part 2, and (c) Part 3.

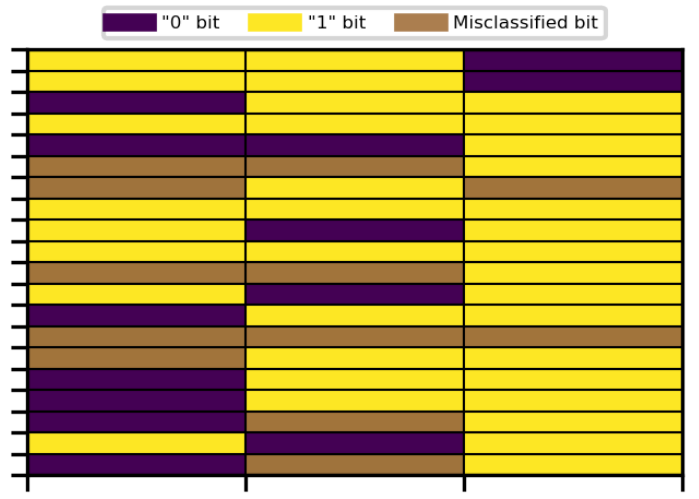

(a)

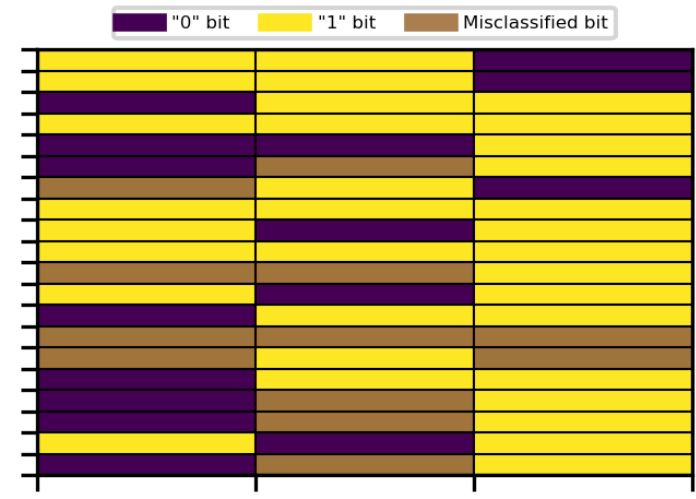

(b)

Figure 7: Part 1 extracted message using (a) Line-based classifier and (b) Area-based classifier.

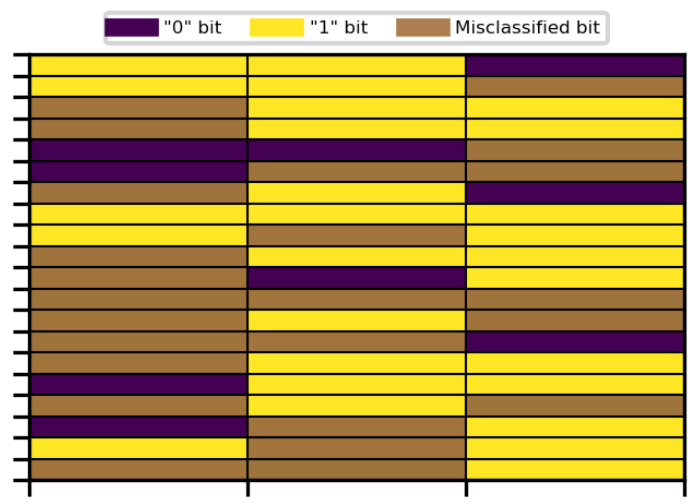

(a)

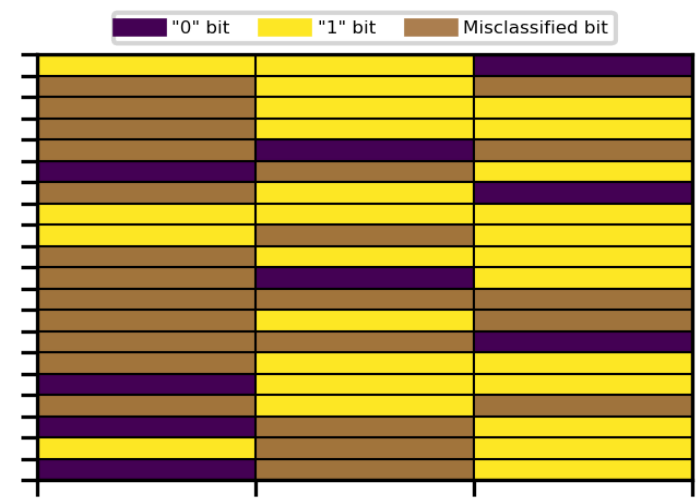

(b)

Figure 8: Part 2 extracted message using (a) Line-based classifier and (b) Area-based classifier. 


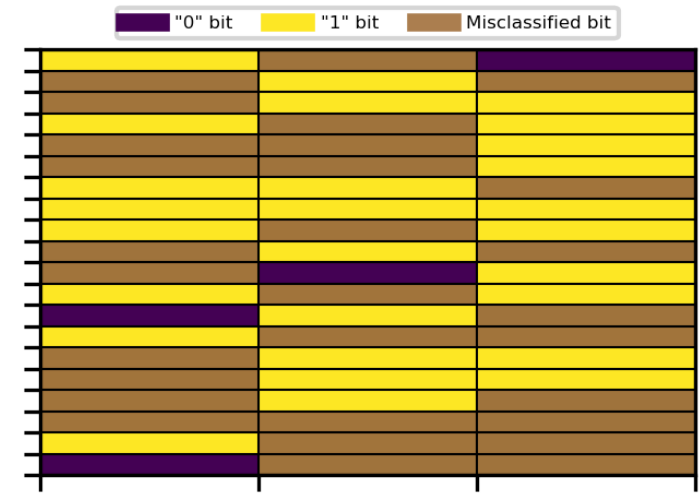

(a)

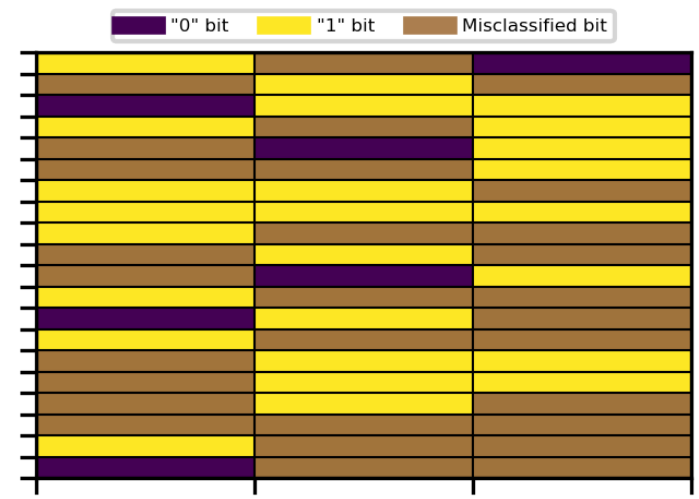

(b)

Figure 9: Part 3 extracted messages using (a) Line-based classifier and (b) Area-based classifier.

Table 2: Confusion matrices for the classifiers and their accuracy

\begin{tabular}{|c|c|c|c|c|c|c|c|c|}
\hline & \multicolumn{4}{|c|}{ Line-based Classifier } & \multicolumn{4}{|c|}{ Area-based Classifier } \\
\hline Part Number & \multicolumn{3}{|c|}{ Confusion Matrix } & Accuracy & \multicolumn{3}{|c|}{ Confusion Matrix } & Accuracy \\
\hline \multirow{3}{*}{$\operatorname{Part} 1(\Delta \mathrm{s}=53 \mathrm{~mm} / \mathrm{s})$} & & Predict "0" & Predict "1" & \multirow{3}{*}{$80 \%$} & & Predict "0" & Predict "1" & \multirow{3}{*}{$80 \%$} \\
\hline & True “0” & $62 \%$ & $38 \%$ & & True “0" & $71 \%$ & $29 \%$ & \\
\hline & True "1" & $10 \%$ & $90 \%$ & & True "1" & $15 \%$ & $85 \%$ & \\
\hline \multirow{3}{*}{ Part $2(\Delta s=32 \mathrm{~mm} / \mathrm{s})$} & & Predict “0" & Predict "1" & \multirow{3}{*}{$60 \%$} & & Predict "0" & Predict “1” & \multirow{3}{*}{$60 \%$} \\
\hline & True "0" & $43 \%$ & $57 \%$ & & True “0” & $43 \%$ & $57 \%$ & \\
\hline & True “1” & $31 \%$ & $69 \%$ & & True "1" & $43 \%$ & $69 \%$ & \\
\hline \multirow{3}{*}{ Part $3(\Delta s=10 \mathrm{~mm} / \mathrm{s})$} & & Predict “0” & Predict “1” & \multirow{3}{*}{$52 \%$} & & Predict “0" & Predict “1” & \multirow{3}{*}{$52 \%$} \\
\hline & True “0” & $19 \%$ & $81 \%$ & & True "0" & $29 \%$ & $71 \%$ & \\
\hline & True "1" & $31 \%$ & $69 \%$ & & True "1" & $36 \%$ & $64 \%$ & \\
\hline
\end{tabular}

[10] Yuki Kubo, Kana Eguchi, Ryosuke Aoki, Shigekuni Kondo, Shozo Azuma, and Takuya Indo. 2019. FabAuth: Printed objects identification using resonant properties of their inner structures. In Extended Abstracts of the 2019 CHI conference on human factors in computing systems. 1-6.

[11] R Leal, FM Barreiros, L Alves, F Romeiro, JC Vasco, M Santos, and C Marto. 2017. Additive manufacturing tooling for the automotive industry. The International Journal of Advanced Manufacturing Technology 92, 5-8 (2017), 1671-1676.

[12] Dingzeyu Li, Avinash S Nair, Shree K Nayar, and Changxi Zheng. 2017. Aircode: Unobtrusive physical tags for digital fabrication. In Proceedings of the 30th annual ACM symposium on user interface software and technology. 449-460.

[13] Zhengxiong Li, Aditya Singh Rathore, Chen Song, Sheng Wei, Yanzhi Wang, and Wenyao Xu. 2018. PrinTracker: Fingerprinting 3D printers using commodity scanners. In Proceedings of the 2018 ACM sigsac conference on computer and communications security. 1306-1323.

[14] Renwei Liu, Zhiyuan Wang, Todd Sparks, Frank Liou, and Joseph Newkirk 2017. Aerospace applications of laser additive manufacturing. In Laser additive manufacturing. Elsevier, 351-371.

[15] David JC MacKay and David JC Mac Kay. 2003. Information theory, inference and learning algorithms. Cambridge university press.

[16] Henrique Teles Maia, Dingzeyu Li, Yuan Yang, and Changxi Zheng. 2019. Lay erCode: optical barcodes for 3D printed shapes. ACM Transactions on Graphics (TOG) 38, 4 (2019), 1-14.

[17] Hao Peng, Peiqing Liu, Lin Lu, Andrei Sharf, Lin Liu, Dani Lischinski, and Baoquan Chen. 2020. Fabricable Unobtrusive 3D-QR-Codes with Directional Light. In Computer Graphics Forum, Vol. 39. Wiley Online Library, 15-27.

[18] Stephen M Pizer, E Philip Amburn, John D Austin, Robert Cromartie, Ari Geselowitz, Trey Greer, Bart ter Haar Romeny, John B Zimmerman, and Karel Zuiderveld. 1987. Adaptive histogram equalization and its variations. Computer vision, graphics, and image processing 39, 3 (1987), 355-368.

[19] Chen Song, Zhengxiong Li, Wenyao Xu, Chi Zhou, Zhanpeng Jin, and Kui Ren 2018. My smartphone recognizes genuine QR codes! practical unclonable qr code via 3D printing. Proceedings of the ACM on Interactive, Mobile, Wearable and Ubiquitous Technologies 2, 2 (2018), 1-20.

[20] Masahiro Suzuki, Pailin Dechrueng, Soravit Techavichian, Piyarat Silapasuphakornwong, Hideyuki Torii, and Kazutake Uehira. 2017. Embedding information into objects fabricated with 3-D printers by forming fine cavities inside them. Electronic Imaging 2017, 7 (2017), 6-9.

[21] Stéfan van der Walt, Johannes L. Schönberger, Juan Nunez-Iglesias, François Boulogne, Joshua D. Warner, Neil Yager, Emmanuelle Gouillart, Tony Yu, and the scikit-image contributors. 2014. scikit-image: image processing in Python. Peerf 2 (6 2014), e453. https://doi.org/10.7717/peerj.453

[22] Geert van Kempen, Michael van Ginkel, Lucas van Vliet, Cris Luengo, and Bernd Rieger. 2021. DIPimage. https://diplib.org/DIPimage.html

[23] Andrew Viterbi. 1971. Convolutional codes and their performance in communication systems. IEEE Transactions on Communication Technology 19, 5 (1971), 751-772.

[24] Chao Wei, Zhe Sun, Yihe Huang, and Lin Li. 2018. Embedding anti-counterfeiting features in metallic components via multiple material additive manufacturing. Additive Manufacturing 24 (2018), 1-12.

[25] Stephen B Wicker and Vijay K Bhargava. 1999. Reed-Solomon codes and their applications. John Wiley \& Sons.

[26] Karl DD Willis and Andrew D Wilson. 2013. Infrastructs: Fabricating information inside physical objects for imaging in the terahertz region. ACM Transactions on Graphics (TOG) 32, 4 (2013), 1-10.

[27] Mark Yampolskiy, Wayne E King, Jacob Gatlin, Sofia Belikovetsky, Adam Brown, Anthony Skjellum, and Yuval Elovici. 2018. Security of additive manufacturing: Attack taxonomy and survey. Additive Manufacturing 21 (2018), 431-457.

[28] Xin Zhang, Qian Wang, Toby Breckon, and Ioannis Ivrissimtzis. 2018. Watermark retrieval from $3 \mathrm{~d}$ printed objects via convolutional neural networks. arXiv preprint arXiv:1811.07640 (2018) 